\title{
A possible role of gas-phase electrophoretic mobility molecular analysis (nES GEMMA) in extracellular vesicle research
}

\author{
Stephanie Steinberger ${ }^{1} \cdot$ Sobha Karuthedom George ${ }^{2} \cdot$ Lucia Lauková $^{2} \cdot$ René Weiss $^{2} \cdot$ Carla Tripisciano $^{2}$. \\ Ruth Birner-Gruenberger ${ }^{1} \cdot$ Viktoria Weber $^{2} \cdot$ Günter Allmaier $^{1} \cdot$ Victor U. Weiss ${ }^{1}$ (D)
}

Received: 30 July 2021 / Revised: 21 September 2021 / Accepted: 23 September 2021 / Published online: 7 October 2021

(C) The Author(s) 2021

\begin{abstract}
The emerging role of extracellular vesicles (EVs) as biomarkers and their envisioned therapeutic use require advanced techniques for their detailed characterization. In this context, we investigated gas-phase electrophoresis on a nano electrospray gas-phase electrophoretic mobility molecular analyzer (nES GEMMA, aka nES differential mobility analyzer, nES DMA) as an alternative to standard analytical techniques. In gas-phase electrophoresis, single-charged, surface-dry, native, polydisperse, and aerosolized analytes, e.g., proteins or bio-nanoparticles, are separated according to their electrophoretic mobility diameter, i.e., globular size. Subsequently, monodisperse particles are counted after a nucleation step in a supersaturated atmosphere as they pass a focused laser beam. Hence, particle number concentrations are obtained in accordance with recommendations of the European Commission for nanoparticle characterization (2011/696/EU from October 18th, 2011). Smaller sample constituents (e.g., copurified proteins) can be detected next to larger ones (e.g., vesicles). Focusing on platelet-derived EVs, we compared different vesicle isolation techniques. In all cases, nanoparticle tracking analysis (NTA) confirmed the presence of vesicles. However, nES GEMMA often revealed a significant co-purification of proteins from the sample matrix, precluding gas-phase electrophoresis of less-diluted samples containing higher vesicle concentrations. Therefore, mainly peaks in the protein size range were detected. Mass spectrometry revealed that these main contaminants belonged to the group of globulins and coagulation-related components. An additional size exclusion chromatography (SEC) step enabled the depletion of co-purified, proteinaceous matrix components, while a label-free quantitative proteomics approach revealed no significant differences in the detected EV core proteome. Hence, the future in-depth analysis of EVs via gas-phase electrophoresis appears feasible.
\end{abstract}

Keywords Gas-phase electrophoresis · Extracellular vesicle · Exosome · nES GEMMA · nES DMA · Mass spectrometry

\section{Introduction}

Extracellular vesicles (EVs) consisting of a lipid bilayer encapsulating an aqueous lumen are bio-nanoparticles secreted by cells. They have been described in various biological fluids, such as urine, blood, and saliva, and are typically defined by a size range of 30-1000 $\mathrm{nm}$ [1]. Originally, EVs were suggested to transport waste material out of cells [2]. Meanwhile, however, there is ample evidence that EVs are

Victor U. Weiss

victor.weiss@tuwien.ac.at

1 Institute of Chemical Technologies and Analytics, TU Wien, Getreidemarkt 9/164 CTA, A-1060 Vienna, Austria

2 Center for Biomedical Technology, Department for Biomedical Research, Danube University Krems, Krems, Austria essential in many physiological processes, including intercellular communication and cellular homeostasis, next to their transport function for proteins, lipids, DNA, and RNA [3, 4]. Furthermore, they play essential roles in immunomodulation, coagulation, and thrombosis, as well as tumor metastasis [5-7]. Depending on the physiological state of their parent cells, their tissue of origin, and their microenvironment, differences in the composition and function of EVs occur [3, 8-10].

Due to their availability in body fluids and their composition that reflects the state of their parent cells, EVs have been suggested as valuable biomarkers [11-14]. However, for this application, the enrichment of EVs out of complex biological matrices and the optimization of corresponding preparation protocols are required. Various analytical strategies have been described for the isolation of EVs from biological fluids or cells to date, including (ultra)centrifugation $[15,16]$, 
chromatographic approaches with a special focus on affinity and size exclusion chromatography (SEC) [17], (ultra)filtration [18], and precipitation [19]. Commercially available EV isolation kits, which combine centrifugation, precipitation, and enzymatic digestion of samples, are widely applied due to their claimed straightforward usage, speed of analysis, and availability [20-22]. However, all these methods share similar limitations regarding EV recovery and purity due to only partial EV enrichment, co-purification of nonvesicular components, and analyte loss based on EV interactions with reagents or supporting materials [23]. The lack of a universal marker for all EV populations often requires multiple purification rounds applying orthogonal methods in order to obtain EV preparations of high purity, which is associated with a loss of EV material.

Besides challenges in EV enrichment, the characterization of these bio-nanoparticles is a challenging task, because vesicle numbers are often low, and native particle sizes are in a range that is not easily targetable. To date, prevalent characterization techniques include flow cytometry and nanoparticle tracking analysis (NTA), often utilized in combination with laser-induced fluorescence, laser light scattering, microscopic techniques, and affinity-based methods [24, 25]. It is of note that some of the aforementioned methods yield information on bio-nanoparticle size and size distribution (e.g., microscopy techniques), however, at the cost of a large number of necessary analyses in order to obtain a sufficiently large, statistically valid dataset. Other methods do not detect smaller sized sample components besides vesicles due to inherent constraints, e.g., characterization techniques based on light scattering. Despite these shortcomings, EV material prepared and characterized in such ways has been applied for comprehensive studies targeting vesicle protein, genome, and lipid content [5, $10,26]$.

In this manuscript, we focus on the applicability of nano electrospray gas-phase electrophoretic mobility molecular analysis (nES GEMMA) as a possible supplement to established analytical techniques for EV characterization. In addition to the acquisition of size distributions of surface-dry analytes and particle counts as suggested by the European Commission for characterization of material in the nanometer size range (2011/696/EU from October 18th, 2011), nES GEMMA offers the possibility of collecting and concentrating EVs on supporting materials. Hence, analyses with orthogonal methods, such as mass spectrometry [27], atomic force microscopy [28], dot blot analysis [29, 30], or cell culture [31] investigations, are feasible. Additionally, analyte molecular weight values can be estimated from nES GEMMA data [32].

nES GEMMA is based on the separation of single-charged nano-sized analytes in the gas phase according to their electrophoretic mobility diameter (EM diameter) and was first described by Kaufman and colleagues in 1996 [33]. Singlecharged nanoparticles are obtained after electrospraying analytes from a volatile electrolyte solution (nES process) with subsequent drying of the EV-containing droplets and charge reduction in a bipolar atmosphere induced by a ${ }^{210} \mathrm{Po}$ $\alpha$-particle source or similar [34-36]. Subsequently, the separation itself occurs inside a nano differential mobility analyzer (nDMA) within a high laminar flow of particle-free, compressed air and an orthogonal, tunable electric field. Variation of the field strength enables particle separationmonomobile analyte fractions are obtained after passage of the nDMA. Subsequently, particles act as nuclei for condensation in a supersaturated atmosphere of n-butanol or water in the ultrafine condensation particle counter (CPC) of the instrument. Particles are counted as they induce laser light scattering. Such a setup is also known under several other namesnES Differential Mobility Analyzer (nES DMA) [37, 38], LiquiScan ES [39], Macro Ion Mobility Spectrometer (MacroIMS) [40], or Scanning Mobility Particle Sizer (SMPS) [41]. However, for matters of consistency with work from our group as well as others, we have chosen to remain with the term nES GEMMA.

Previous studies have demonstrated the applicability of nES GEMMA for the characterization of liposomesnanovesicles consisting of a lipid bilayer with an aqueous core - in terms of vesicle size and heterogeneity of preparations [42]. In addition, a first study already reported nES GEMMA also for EVs [43]. However, in this study, only particles exceeding $20 \mathrm{~nm}$ EM diameter (> 1.5 MDa on the protein scale; [32]) were analyzed with adjusted instrument settings. Furthermore, nES GEMMA has already been successfully applied for lipoprotein particle characterization [42, 44, 45].

nES GEMMA enables to analyze a size range from single digit up to several hundred nanometers, and instrument settings have to be adapted for smaller and larger sample components (refer to Supplementary Information Figure S1 for a schematic overview on the targetable analyte size range via nES GEMMA and NTA, respectively). In order to analyze smaller sized analytes such as small proteins, a high sheath flow inside the nDMA yields highly resolved peaks. At the same time, however, the targetable size range of bionanoparticles is significantly reduced. A large size range, on the other hand, comes at the cost of poor peak resolution for homogeneous sample components, such as proteins. In the case of heterogeneous analytes such as intact EVs, no additional peak broadening occurs for low sheath flow values.

Focusing on EV-containing samples purified from complex matrices, this results in two possible analysis approaches especially when taking concentration differences between smaller and larger sample components into consideration. nES GEMMA can either focus on analytes up to a few $10 \mathrm{~nm}$ EM diameter (proteins, genomic material, etc.) with good resolution, or on EVs, excluding the lower size range of the spectrum for analysis ([43]). Concentrating on the first 
approach, it was the aim of our study to demonstrate that $\mathrm{nES}$ GEMMA yields valuable information concerning the purity of extracellular vesicle-containing samples, especially in the region below $20 \mathrm{~nm}$ EM diameter. Particles in this size/ molecular weight range are possibly overlooked when relying on other analysis methods like NTA alone usually enabling detection of particles larger than several $10 \mathrm{~nm}$ hydrodynamic diameter. Especially for proteomics and immunoanalytical approaches targeting EV-associated proteins, sample purity and protein co-purification are crucial, and we suggest that nES GEMMA can contribute significantly to the quality control of EV-containing samples.

\section{Materials and methods}

\section{Isolation of extracellular vesicles}

Medical grade platelet concentrates from healthy donors were applied for the isolation of EVs. Concentrates were obtained from the Clinic for Blood Group Serology and Transfusion Medicine (Medical University Vienna, Vienna, Austria) as approved by the Ethics committee of Danube University Krems (Krems, Austria) (ethics votum number ECS2177/ 2015). Written informed consent was obtained from all donors in our study. EVs were isolated either using the "Total Exosome Isolation Kit from plasma" (Invitrogen, Carlsbad, CA, USA) based on a low-speed centrifugation step after EV precipitation or a two-step ultracentrifugation approach at $2 \times 10^{4} \mathrm{~g}$ and $1 \times 10^{5} \mathrm{~g}$, respectively [46]. Prior to ultracentrifugation, platelets were removed from samples at $2.5 \times$ $10^{3} \mathrm{~g}$. Following ultracentrifugation, the pellets were resuspended in phosphate-buffered saline (PBS; Life Technologies, Paisley, UK) [46]. PBS was filtered $(0.1 \mu \mathrm{m}$ Minisart syringe filter, Sartorius Stedim Biotech, Goettingen, Germany) prior to use.

In an additional approach, EVs after ultracentrifugation (500 $\mu \mathrm{L}$ volume) were further processed using size exclusion chromatography (qEV, Izon Science, Burnside, Christchurch, New Zealand). Fractions (500 $\mu \mathrm{L}$ each) were collected and fractions containing Annexin V-positive $\left(\mathrm{AnxV}^{+}\right) \mathrm{EVs}$ according to flow cytometry were pooled and centrifuged at 2 $\times 10^{4} \mathrm{~g}$ and $1 \times 10^{5} \mathrm{~g}$, respectively, resulting in an EV20k SEC and an EV100k SEC sample.

\section{Flow Cytometric characterization of platelet-derived EVs}

EV suspensions were diluted in PBS to a protein concentration of $1 \mu \mathrm{g} \mathrm{mL}^{-1}$. Aliquots of $100 \mu \mathrm{L}$ were stained for $15 \mathrm{~min}$ at RT in the dark with fluorescein isothiocyanate-conjugated Annexin V (Becton Dickinson, Eysins, Switzerland) as marker of phosphatidylserine, as well as with phycoerythrin cyanin
7 (PE-PC7)-conjugated anti-CD41 antibody (Beckman Coulter, Brea, CA, USA) as platelet marker. All antibody conjugates were centrifuged at $1.7 \times 10^{4} \mathrm{~g}$ for $10 \mathrm{~min}$ at ambient temperature prior to use to remove aggregates. Stained samples were further diluted fivefold in PBS and analyzed on a CytoFLEX LX flow cytometer (Beckman Coulter). Fluorescent-green silica particles $(1 \mu \mathrm{m}, 0.5 \mu \mathrm{m}, 0.1 \mu \mathrm{m}$; excitation/emission 485/510 nm; Kisker Biotech, Steinfurt, Germany) were used for calibration. The triggering signal was set to violet side scatter and the EV gate was set as shown previously [46-48]. Data were analyzed using the Kaluza Software (Beckman Coulter).

\section{nES GEMMA measurements}

Measurements on a nES GEMMA require analytes dissolved in a volatile electrolyte solution. Therefore, PBS of EV preparations was exchanged to $40 \mathrm{mM}$ ammonium acetate, $\mathrm{pH} 8.4$ (Sigma-Aldrich, St. Louis, MO, USA) with 10k MWCO filters (Pall Laboratory, Port Washington, NY, USA) at $9.3 \times$ $10^{3} \mathrm{~g}$. Subsequently, the EV-containing samples were further diluted 1:10 (v:v) by means of an ammonium acetate $(40 \mathrm{mM}$, $\mathrm{pH}$ 8.4) solution. The $\mathrm{nES}$ occurred at constant pressure of 4 psid $(0.28 \mathrm{bar})$ and in average $2.0 \mathrm{kV}$ spray voltage at the tip of a $25-\mu \mathrm{m}$-inner diameter, cone-tipped capillary [49] in the nES aerosol generator (model 3480, TSI Inc, Shoreview, MN, USA). Subsequently, a gas flow of $1.0 \mathrm{~L} \mathrm{~min}^{-1}$ dried air and $0.1 \mathrm{~L} \mathrm{~min}^{-1}$ of carbon dioxide was used to transport the polydisperse aerosol through the bipolar atmosphere inside the charge reduction chamber induced by a ${ }^{210} \mathrm{Po} \alpha$-particle source. Concomitantly, the electrolyte solution evaporated and single-charged, surface-dry analytes were separated in a nano differential mobility analyzer (nDMA, model 3080, TSI Inc) applying a sheath flow of $8.0 \mathrm{~L} / \mathrm{min}$. Following separation, nanoparticles were counted by laser light scattering after having induced nucleation in a n-butanol saturated atmosphere in an ultrafine condensation particle counter (model 3776, TSI Inc). The measuring range encompassed particles from 3.0 to $91.4 \mathrm{~nm}$ EM diameter. Five scans (adjustment of the nDMA separation voltage- $190 \mathrm{~s}$ scan time, $20 \mathrm{~s}$ for voltage re-setting) were combined via their median to obtain a corresponding nES GEMMA spectrum.

\section{Nanoparticle tracking analysis (NTA)}

For NTA, the samples were diluted 1:10,000 (v:v) and 1:50,000 (v:v), for SEC samples 1:1000 (v:v) in high-purity water $\left(18.2 \mathrm{M} \Omega \mathrm{cm}\right.$ resistivity at $25{ }^{\circ} \mathrm{C}$, MilliQ-System, Merck, Darmstadt, Germany), or $40 \mathrm{mM}$ ammonium acetate solution, $\mathrm{pH} 8.4$ and immediately measured after dilution. The measurements were performed with a Zetaview PMX120 (ParticleMetrix, Meerbusch, Germany) at $22{ }^{\circ} \mathrm{C}$. Each measurement consists of 11 scattering measurement positions. 
The minimum brightness of the expected particles was set to auto, the particle size range was set to $5-200 \mathrm{~nm}$, the shutter was set to 100 , the minimum tracelength was set to 15 , and the frame rate was set to 15 (arbitrary units, each). On average, the concentration of EV samples was $10^{11}-10^{12}$ particles per milliliter, while SEC samples only contained around $10^{8}-10^{9}$ particles per milliliter. The obtained data was analyzed with the corresponding instrument software (ZetaView 8.05.05 SP2) to calculate mean, standard deviation, and size distribution.

\section{MALDI MS}

One approach to identify proteins in EV20k and EV100k samples was based on SDS-PAGE, in-gel tryptic digest, and MALDI MS. A NuPAGE (Invitrogen, Waltham, MA, USA) $12 \%$ Bis-Tris gel electrophoresis with a sample concentration of $0.5 \mu \mathrm{g}$ total protein content per lane was performed, using pre-stained molecular weight markers (NovexSharp prestained, Invitrogen, Waltham, MA, USA) followed by a MS-compatible silver staining based on [50]. Visible protein bands were excised and subjected to an overnight tryptic ingel digest [51]. For a better digestion efficiency, a trypsin/lysC mixture with the concentration of $0.1 \mathrm{ng} / \mu \mathrm{L}$ from Promega (Madison, WI, USA) was used. In addition, the digested peptides were purified with a ZipTip $_{\mathrm{C} 18}$ (Merck) purification protocol. The purified peptides were directly eluted with a $3 \mathrm{mg} \mathrm{mL}^{-1} \alpha$-cyano-4-hydroxycinammic acid (CHCA) matrix (acetonitrile/water/TFA (60:40:0.1) (v:v:v)) solution on a MTP 384 stainless steel MALDI MS target. All measurements were performed on an UltrafleXtreme mass spectrometer (Bruker Daltonics, Bremen, Germany) in positive RTOF (reflector time-of-flight) and tandem TOF/RTOF mode. For the acquisition of the mass spectra in the RTOF and tandem TOF/ RTOF mode, an accumulation of 6000 and 10,000 laser shots, respectively, at a laser power of $39 \%$ and $1 \mathrm{kHz}$ in a range of $500-3500 \mathrm{~m} / \mathrm{z}$ was performed. The obtained data was analyzed with the manufacturer's software flexAnalysis (v.3.4 Build 57) and the protein identification was implemented with the Mascot Search Engine (MatrixScience, London, UK). As a database, the NCBI protein database for Homo sapiens was chosen, as well as carbamidomethylation on Cys as a fixed modification. Acetylation on the protein $\mathrm{N}$-terminus, oxidation on Met, phosphorylation on Ser and Thr, and deamidation on Asn and Gln were set as variable modifications. Furthermore, the missed cleavages were set to 2, the molecular mass tolerance to $\pm 0.3 \mathrm{Da}$, and the fragment ion tolerance to $\pm 0.5 \mathrm{Da}$.

\section{LC-ESI-MS}

Three independent preparations of EV samples before and after SEC corresponding to 600 -ng protein content were subjected to clean up via SDS-PAGE, reduction, alkylation, and in-gel tryptic digest. One-third of the digests was analyzed by LC-MS/MS. Chromatography was carried out on an Ultimate 3000 RCS Nano Dionex system (Thermo Scientific, Waltham, MA, USA) equipped with an Ionopticks Aurora Series UHPLC C18 column $(250 \mathrm{~mm} \times$ $75 \mu \mathrm{m}, 1.6 \mu \mathrm{m}$ ) (Ionopticks, Parkville, Australia). Solvent A was $0.1 \%$ formic acid in water and solvent $\mathrm{B}$ acetonitrile containing $0.1 \%$ formic acid. A total LC-MS/MS run per sample lasted for $136.5 \mathrm{~min}$ with the following gradient: $0-5.5 \mathrm{~min}$ : 2\% B; 5.5-65.5 min: $2-17 \%$ B; $65.5-95.5$ min: $25-37 \%$ B; 105.5-115.5 min: $37-95 \%$ B; $115.5-125.5 \mathrm{~min}: 95 \% \mathrm{~B}$; 125.5-126.5 min: $95-2 \% \mathrm{~B} ; 126.5-136.5 \mathrm{~min}: 2 \% \mathrm{~B}$ at a flow rate of $400 \mathrm{nl} \mathrm{min}^{-1}$ and a column temperature of $50{ }^{\circ} \mathrm{C}$. The timsTOF mass spectrometer (Bruker Daltonics) was operated in positive ion mode with enabled trapped ion mobility spectrometry (TIMS) at 100\% duty cycle (100-ms cycle time). Scan mode was set to parallel accumulation-serial fragmentation (PASEF) for the scan range of $100-1700 \mathrm{~m} / z$. Source capillary voltage was set to $1500 \mathrm{~V}$ and dry gas flow to $3 \mathrm{~L} \mathrm{~min}^{-1}$ at $180{ }^{\circ} \mathrm{C}$. LC-MS/MS data was subjected to MaxQuant (v1.6.17.0) [52-54] Andromeda search of the Uniprot human database containing common contaminants $(20,467$ entries) using 1\% PSM (peptide spectrum matches) and protein FDR (false discovery rate) as threshold for identification (including carbamidomethylation on Cys as fixed, oxidation on Met, and acetylation on protein $\mathrm{N}$-terminus as variable modifications, minimum peptide length 7 amino acids) and minimum 2 ratios of unique and razor peptides for label-free quantification (LFQ).

LFQ values were $\log 2$ transformed and subjected to statistical analysis using Perseus (v1.6.12.0.) [55]. Prior to statistical testing, the matrix was filtered for common contaminants and to keep only those proteins with reported valid values in at least 3 samples in at least one group. Missing values were then imputed from a normal distribution with a width of 0.3 and a downshift of 1.8. Histograms of intensities of quantified proteins are shown in Supplementary Information Figure S2 suggesting a normal distribution, where measured proteins are depicted in blue while imputed proteins are depicted in red. A two-samplet-test corrected for multi-testing was performed between the groups (permutation-based FDR 5\%, S0 = 2). The MS proteomics datasets were deposited to the ProteomeXchange Consortium via the PRIDE partner repository [56] with the dataset identifier PXD024760 (Reviewer account details: Username: reviewer_pxd024760@ebi.ac.uk; Password: pGPaJoKy).

\section{Results and discussion}

Comprehensive EV research relies on well-characterized, highly purified vesicle material. Focusing on two preparation 
and enrichment techniques - ultracentrifugation and application of an EV precipitation kit-we started our work dealing with EVs purified from human medical platelet concentrate. Enriched EV fractions were characterized using NTA, which detected particles of approximately $120 \mathrm{~nm}$ (centrifugation) and $160 \mathrm{~nm}$ (precipitation) hydrodynamic diameter (Fig. 1A). However, subjecting the corresponding samples to gas-phase electrophoresis revealed the presence of material in the EM diameter range, which was associated with free proteins at very high concentrations. In contrast, signals for larger assemblies (EVs) were very low (Fig. 1B) and the occurrence of free proteins in such high concentrations precluded nES GEMMA of less-diluted samples which would putatively have been led to increased particle counts in the higher EM diameter range.

Focusing on the origin of proteins detected via nES GEMMA, we reasoned that these either might be co-purified with EVs from solution or might originally be EV-associated (attached to the EV surface, encapsulated in the vesicle aqueous core, or were a part of the lipid EV bilayer). In the latter case, detection of free proteins in such high concentrations especially in combination with low signals obtained in the higher EM diameter range probably indicates loss of EV integrity during sample preparation.

In order to analyze samples via nES GEMMA, the removal of non-volatile sample buffer components is a prerequisite. In this context, interaction of vesicles with membrane material applied for electrolyte exchange, the forces exerted on EVs during this process, or electrolyte-dependent changes in osmolarity or $\mathrm{pH}$ might have led to vesicle damage. Thus, EVs would have been no longer detectable due to their removal from samples. As a consequence, proteins recorded in $\mathrm{nES}$ GEMMA spectra would have been released from vesicles upon rupture. On the other hand, sample preparation and nES GEMMAs of intact liposomes as well as other noncovalently bound macromolecule assemblies, such as glycoproteins and lectins, have already been published [30, 42, 45,
57-59]. Therefore, analyte transition from the liquid to the gas phase in nES GEMMA is known to occur under native conditions. Hence, the idea that protein signals detected with $\mathrm{nES}$ GEMMA originated from material released from EVs upon vesicle rupture during storage and electrolyte exchange appeared unlikely. Nevertheless, this possibility was further investigated.

Taking corresponding samples to our NTA setup revealed that EVs were present after the necessary exchange of electrolyte solutions, although reduced in overall particle numbers by about $90 \%$ as deduced from NTA measurements. Particle hydrodynamic diameter values remained comparable prior to and after exchange of electrolyte solutions possibly excluding preferential loss of vesicle subclasses (Fig. 1C; based on different sample dilutions in order to obtain comparable analyte numbers).

Based on this observation, the idea of co-purification of proteins during the enrichment of EVs gained significance. Such a co-purification would usually go unnoticed, as most analysis methods other than nES GEMMA concentrate on a size region of EVs rather than focusing on co-purified proteins (refer to Supplementary Information Figure S1 for a schematic comparison of the nES GEMMA and NTA sizing range). It was thus our intention to demonstrate such a co-purification via additional purification steps, after a detailed analysis of copurified sample components.

\section{Identification of main protein components of EV preparations}

During EV enrichment via ultracentrifugation, at $2 \times 10^{4} \mathrm{~g}$, larger vesicle components (EV20k, "microvesicles") are pelleted, whereas at $1 \times 10^{5} \mathrm{~g}$, smaller sized material (EV100k, "exosomes") is obtained. Following these enrichment steps, samples were characterized using NTA and nES GEMMA (Fig. 2A and B), as presented in the previous section. Additionally, proteinaceous sample compounds were

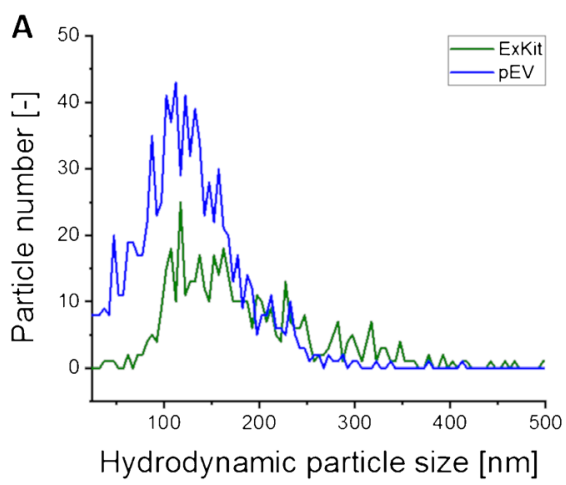

Fig. 1 Comparison of two EV isolation techniques and investigation of the impact of solvent exchange on particle numbers. Comparison of the hydrodynamic particle size distribution (NTA-derived, A) and the dry particle size distribution (nES GEMMA-derived, B) of EVs obtained
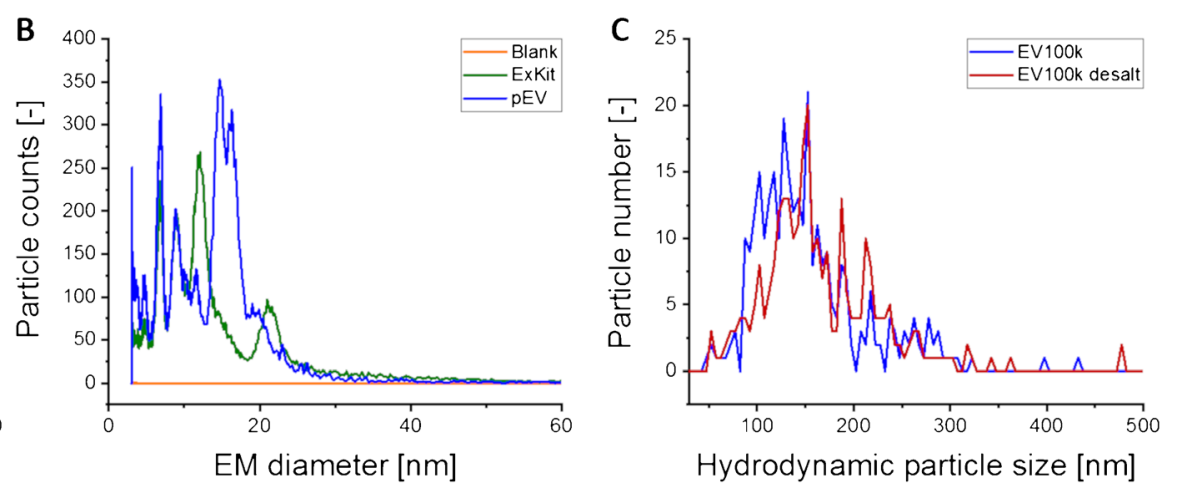

applying two isolation techniques - ultracentrifugation $(\mathrm{pEV})$ and an exosome isolation kit (ExKit). The effect of electrolyte exchange via a $10-\mathrm{kDa}$ MWCO filter $(\mathbf{C})$ on the hydrodynamic size distribution and particle number of the samples, as evaluated by NTA 

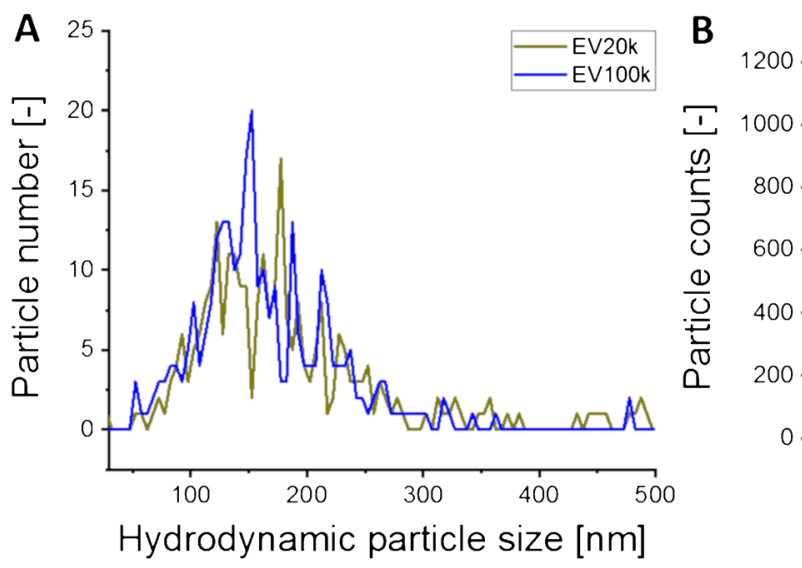

Fig. 2 Characterization of larger (EV20k) and smaller (EV100k) EVs obtained via centrifugal isolation. Hydrodynamic particle size distribution (NTA-derived, A) and dry particle size distribution (nES GEMMA-derived, B) of the EV-containing fractions were measured. SDS-PAGE (C)

analyzed using SDS-PAGE (Fig. 2C), a subsequent tryptic in-gel digestion, and MALDI MS/MS identification. As expected, SDS-PAGE disclosed a multitude of proteins contained in EV samples. Lanes were excised and an in-gel digestion was carried out overnight prior to Zip Tip purification and mass spectrometric analysis by means of a MALDI-TOF/RTOF-MS instrument. Such an approach identified three protein components with $95 \%$ confidence - $\beta$-actin (like) protein (Supplementary Information Figure S3A), haemoglobin (Supplementary Information Figure S3B), and $\alpha$-2-macroglobulin(Supplementary Information Figure S3C). These proteins are already described in ExoCarta (http://www.exocarta.org, date of retrieval: 15.02. 2021) as being associated with platelet-derived EVs. However, these proteins are also blood compounds involved in immune response, coagulation, and platelet function $[26,60,61]$. Thus, whether these proteins were originally EV-associated

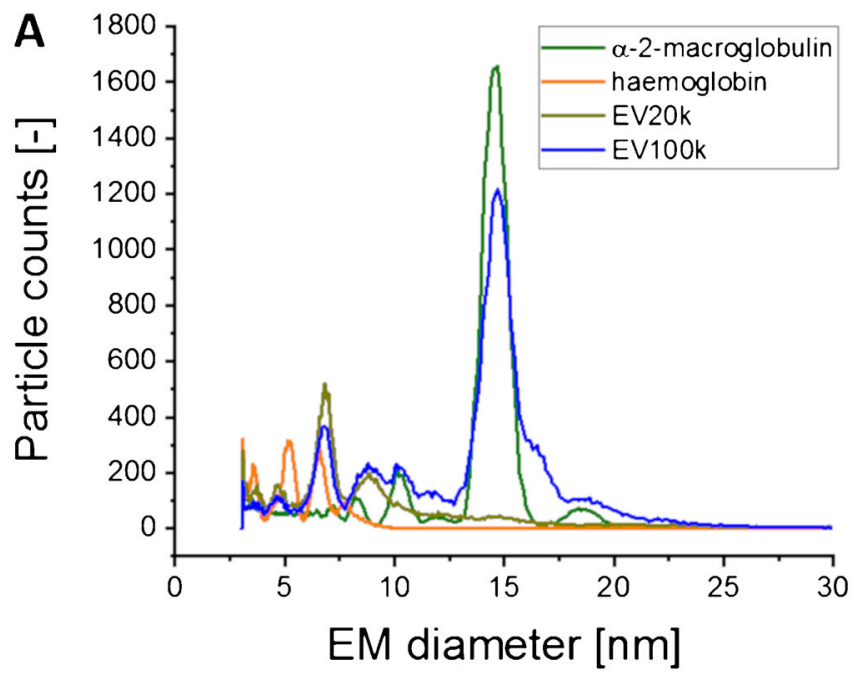

Fig. 3 Relation of nES GEMMA spectra of EVs and identified contaminants. Two contaminants, $\alpha$-2-macroglobulin and haemoglobin, common in human blood and identified via SDS-PAGE, in-gel digest,

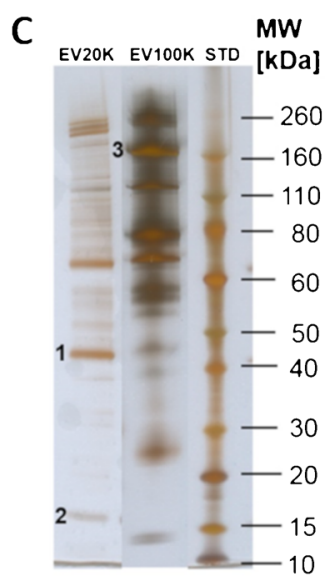

for further analysis with MALDI MS/MS revealed differences in the protein composition of the fractions in comparison to a protein standard (STD)

(encapsulated, part of the lipid bilayer or membrane attached) or were just co-purified still remained elusive via our chosen analytical approach.

Next, to demonstrate that haemoglobin and $\alpha-2$ macroglobulin were part of nES GEMMA spectra, we analyzed these proteins both individually and in a mixture via gas-phase electrophoresis. Originally according to Bacher et al. [32], the molecular weight of proteins correlates with their EM diameter, resulting in a theoretical EM diameter for haemoglobin (hetero-dimer, approx. $64 \mathrm{kDa}$ ) and $\alpha-2$ macroglobulin (homo-tetramer, approx. $720 \mathrm{kDa}$ ) of $6.7 \mathrm{~nm}$ and $14.9 \mathrm{~nm}$, respectively. Measurements of both proteins with nES GEMMA led to experimentally determined EM diameters of $6.6 \pm 0.3 \mathrm{~nm}$ and $14.6 \pm 0.2 \mathrm{~nm}(n=3$ measurements), respectively, which corresponds well with peaks in the pattern of the EV-containing samples (Fig. 3).

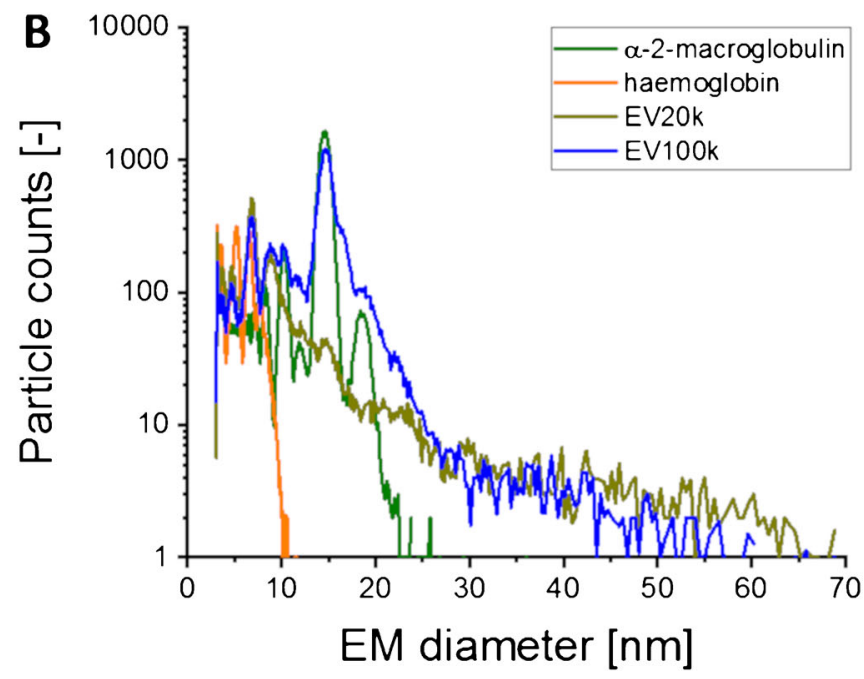

and MALDI MS/MS, were compared to EV isolates. Data is shown in linear scale (A) and logarithmic scale (B) to emphasize nES GEMMA signals obtained for vesicles in the higher EM diameter range 


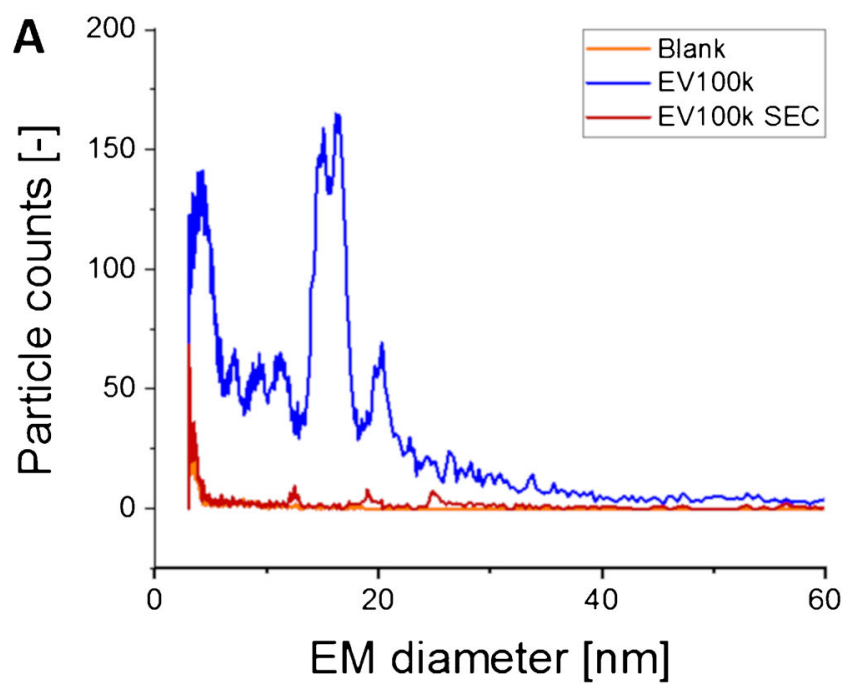

Fig. 4 Influence of SEC on EV preparations obtained after ultracentrifugation. The dry particle size distribution (nES GEMMAderived, A) and the hydrodynamic particle size distribution (NTA-

\section{Depletion of proteinaceous sample components and its effect on a proteomics profiling approach}

As the high concentration of proteinaceous material in EV samples caused considerable problems in comprehensive vesicle analysis, SEC was introduced as an additional purification step after ultracentrifugation. Focusing on EV100k preparations, a comparison between ultracentrifugation alone and a

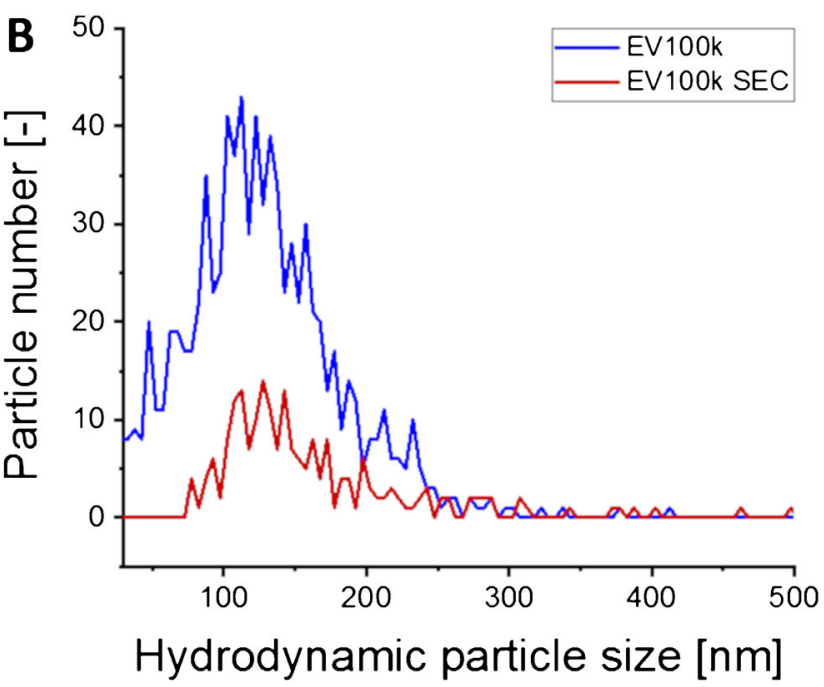

derived, B) of smaller EVs (EV100k) were measured and reveal loss of contaminating material in the low EM diameter range as well as a reduction of detected vesicles

combination of ultracentrifugation and SEC clearly indicated a depletion of low EM diameter material in nES GEMMA (Fig. 4A) following SEC. At the same time, NTA confirmed the presence of EVs after the additional SEC step, although a loss of vesicles by about $60 \%$ (particle number based) occurred, which was possibly due to vesicle interaction with the chromatographic material and/or shear forces within the separation column (Fig. 4B). This finding renders ruptured
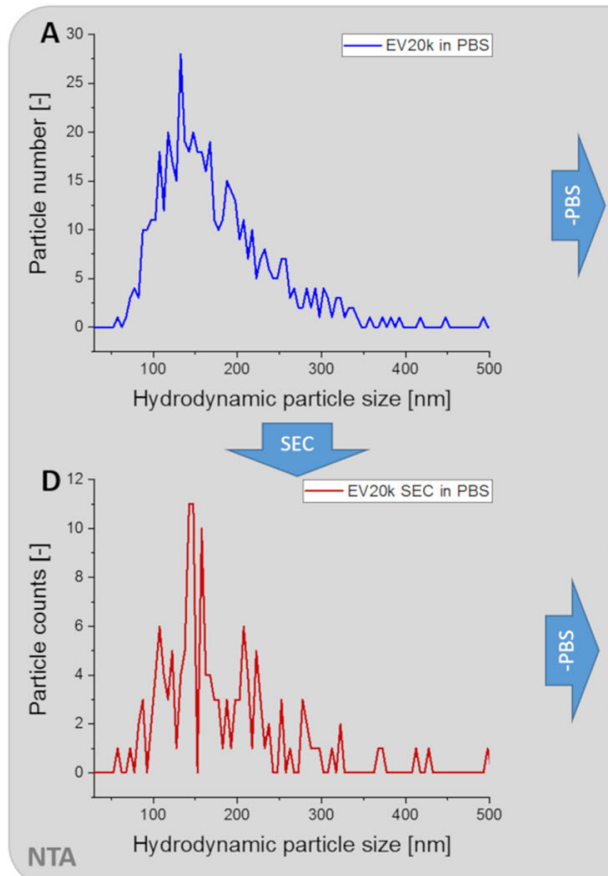

Fig. 5 Influence of solvent exchange via filtration on EV samples. The hydrodynamic size distribution was measured before and after the exchange of PBS to an ammonium acetate solution (A and $\mathbf{B} ; \mathbf{D}$ and $\mathbf{E}$ )

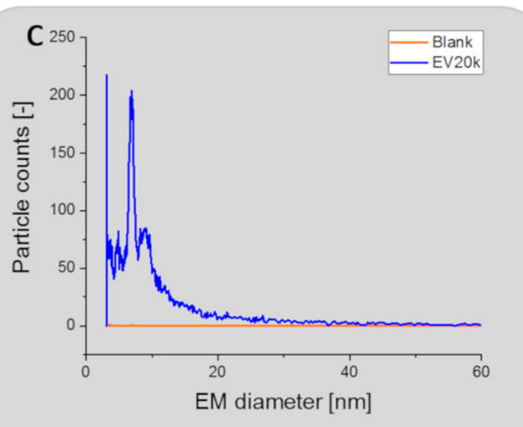

SEC

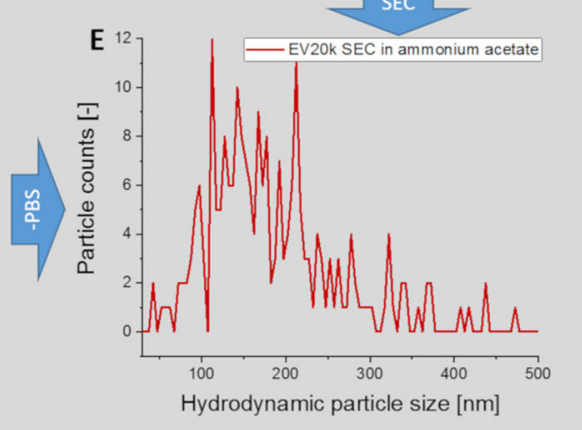

for the larger EVs before and after SEC. The corresponding nES GEMMA measurements $(\mathbf{C}, \mathbf{F})$ display the dry particle size distribution 
vesicles as the origin of detected proteins highly improbable, as in this case also protein peaks should have been detected in the last nES GEMMA sample of the described workflow.

An additional complete dataset demonstrating our hypothesis of co-purifed proteins detected in the low EM diameter range upon nES GEMMA is presented in Fig. 5. Presence of EVs in samples is confirmed via NTA measurements. Exchange of the electrolyte solution to ammonium acetate resulted in a reduction of vesicle counts by $\geq 60 \%$, as demonstrated by NTA. At the same time, protein-related peaks were detectable by means of nES GEMMA. Processing the original sample via SEC resulted likewise in a loss of vesicles by about $60 \%$. However, EV occurrence could still be confirmed by NTA measurements. Another step of electrolyte exchange of this SEC purified sample also led to vesicle loss as detected by NTA measurements. However, at the same time, nES GEMMA failed to detect protein-related peaks.

We next analyzed whether additional sample pretreatment steps such as SEC would significantly influence the protein content of EVs. Focusing on an EV preparation not showing any co-purified proteins in the low EM diameter range via nES GEMMA, we compared LC-MS/ MS data of a sample with and without additional SEC, employing three technical replicates of the preparations (samples Tech 1-Tech 3). We identified and quantified between 2182 and 2217 proteins in each sample when injecting similar peptide amounts. Overall, 1968 proteins were common in all 6 samples; 123 proteins in 5, 30 proteins in 4 , and 8 proteins in only 3 samples after filtering for at least three valid values in at least one group. As demonstrated in Fig. 6, no significant difference in detected proteins (overall $n=2129$ ) could be obtained before and after SEC. The complete list of proteins can be found in Supplementary Information Table S1. Moreover, about 2000 common proteins in the individual preparations (sample Tech 1: 2054; sample Tech 2: 2057; sample Tech 3: 2069) before and after SEC followed a perfect linear correlation.

Hence, we reasoned that application of SEC is a suitable method for reduction of co-purified proteinaceous sample components while vesicle-associated proteins are not affected by SEC, despite a significant loss of overall vesicle numbers.
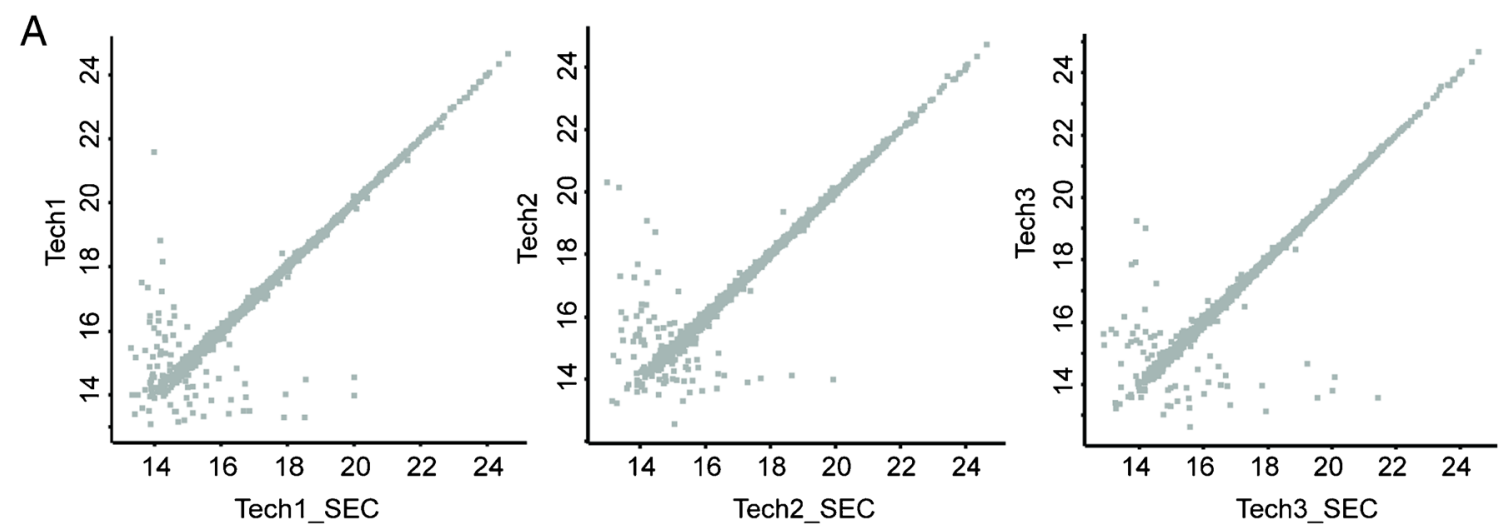

B

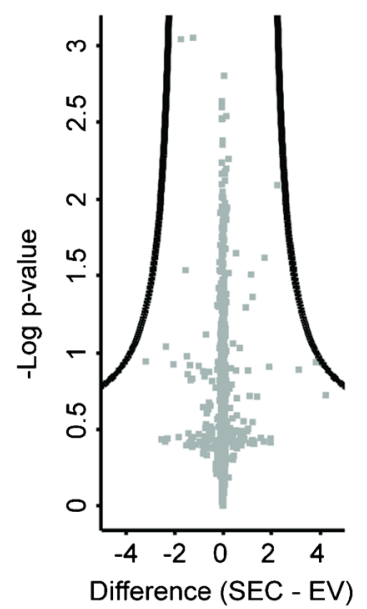

Fig. 6 LC-ESI-MS proteomics analysis of smaller EVs (100 k) before and after SEC isolation. A Scatter plot of three independent EV preparations (named Tech 1, Tech 2, Tech 3) before and after SEC shows that $\log 2$ LFQ intensities of common proteins depict a highly linear correlation (A). B Volcano plot of EV samples before (EV) and after SEC (SEC) depicts $-\log p$ values after multi-testing control (FDR 5\%) of a twosided $t$-test $(\mathrm{S} 0=2)$ versus the differences between mean $\log 2 \mathrm{LFQ}$ intensities before and after SEC, respectively. Samples show a high similarity with no significant difference in the proteome of the EVs due to the application of an additional SEC isolation step (B) 


\section{Conclusion}

The heterogeneity of EVs makes their isolation, purification, and characterization a challenging task. Characterization of EVs with nES GEMMA is an alternative option in addition to current characterization techniques, such as flow cytometry, NTA, laser light scattering, microscopic techniques, and affinity-based methods [24, 25]. Applying nES GEMMA, we observed co-purification of proteins in high quantities, adding additional complexity to sample characterization. The main coenriched components were identified via a SDS-PAGE, in-gel digest, and MALDI MS/MS approach. nES GEMMA measurements corroborated the MS-based protein identification.

Previous studies have questioned the influence of SEC on vesicle membrane integrity and surface protein patterns $[26$, 55]. Using nES GEMMA, we found that an additional SEC step following EV isolation by ultracentrifugation depleted the putatively co-isolated proteins. At the same time, SEC appears to have no influence on the $\mathrm{EV}$ proteome as such, even though it leads to a loss of approximately $60 \%$ of EVs as determined via NTA.

To conclude, nES GEMMA is a valuable approach for quality control of EV-containing samples under native conditions, as it allows for the detection of co-purified proteins from complex matrices.

Availability of data and material The data that support the findings of this study are available from the corresponding author upon reasonable request.

Author contribution Initial idea: VUW, CT, VW, GA. Experimental work: SS, SKG, LL, RW, RBG; EV isolation: SKG. Guidance: VUW, CT, VW, GA. Instrumentation: VW, GA, RBG. Funding: VUW, CT, GA, VW, RBG. All authors contributed to the manuscript.

Funding Open access funding provided by TU Wien (TUW). This work was supported by the NÖ Forschungs- und Bildungsges.m.b.H (NFB) (project LSC16-018). This work also received financial support from TU Wien Bibliothek through its Open Access Funding programme.

\section{Declarations}

Ethics approval EV-containing samples were obtained from the Clinic for Blood Group Serology and Transfusion Medicine (Medical University Vienna, Vienna, Austria) as approved by the Ethics committee of Danube University Krems (Krems, Austria) (ethics votum number ECS2177/2015)

Consent to participate Written informed consent was obtained from all EV donors in our study.

Consent for publication All authors consented to the manuscript.

Conflict of interest The authors declare no competing interests.

Open Access This article is licensed under a Creative Commons Attribution 4.0 International License, which permits use, sharing, adaptation, distribution and reproduction in any medium or format, as long as you give appropriate credit to the original author(s) and the source, provide a link to the Creative Commons licence, and indicate if changes were made. The images or other third party material in this article are included in the article's Creative Commons licence, unless indicated otherwise in a credit line to the material. If material is not included in the article's Creative Commons licence and your intended use is not permitted by statutory regulation or exceeds the permitted use, you will need to obtain permission directly from the copyright holder. To view a copy of this licence, visit http://creativecommons.org/licenses/by/4.0/.

\section{References}

1. Colombo M, Raposo G, Thery C. Biogenesis, secretion, and intercellular interactions of exosomes and other extracellular vesicles. Annu Rev Cell Dev Biol. 2014;30:255-89.

2. Johnstone RM, Adam M, Hammond JR, Orr L, Turbide C. Vesicle formation during reticulocyte maturation - association of plasmamembrane activities with released vesicles (exosomes). J Biol Chem. 1987;262:9412-20.

3. Raposo G, Stoorvogel W. Extracellular vesicles: exosomes, microvesicles, and friends. J Cell Biol. 2013;200:373-83.

4. Fevrier B, Raposo G. Exosomes: endosomal-derived vesicles shipping extracellular messages. Curr Opin Cell Biol. 2004;16:415-21.

5. van der Pol E, Boing AN, Harrison P, Sturk A, Nieuwland R. Classification, functions, and clinical relevance of extracellular vesicles. Pharmacol Rev. 2012;64:676-705.

6. Bobrie A, Colombo M, Raposo G, Thery C. Exosome secretion: molecular mechanisms and roles in immune responses. Traffic. 2011;12:1659-68.

7. Becker A, Thakur BK, Weiss JM, Kim HS, Peinado H, Lyden D. Extracellular vesicles in cancer: cell-to-cell mediators of metastasis. Cancer Cell. 2016;30:836-48.

8. Colombo M, Moita C, van Niel G, Kowal J, Vigneron J, Benaroch P, Manel N, Moita LF, Thery C, Raposo G. Analysis of ESCRT functions in exosome biogenesis, composition and secretion highlights the heterogeneity of extracellular vesicles. J Cell Sci. 2013;126:5553-65.

9. van Niel G, D'Angelo G, Raposo G. Shedding light on the cell biology of extracellular vesicles. Nat Rev Mol Cell Bio. 2018;19:213-28.

10. Kowal J, Arras G, Colombo M, Jouve M, Morath JP, PrimdalBengtson B, Dingli F, Loew D, Tkach M, Thery C. Proteomic comparison defines novel markers to characterize heterogeneous populations of extracellular vesicle subtypes. P Natl Acad Sci USA. 2016;113:E968-77. https://doi.org/10.1073/pnas.1521230113.

11. Gyorgy B, Szabo TG, Pasztoi M, Pal Z, Misjak P, Aradi B, Laszlo V, Pallinger E, Pap E, Kittel A, Nagy G, Falus A, Buzas EI. Membrane vesicles, current state-of-the-art: emerging role of extracellular vesicles. Cell Mol Life Sci. 2011;68:2667-88.

12. EL Andaloussi S, Maeger I, Breakefield XO, Wood MJA. Extracellular vesicles: biology and emerging therapeutic opportunities. Nat Rev Drug Discov. 2013;12:348-58.

13. Lee Y, El Andaloussi S, Wood MJA. Exosomes and microvesicles: extracellular vesicles for genetic information transfer and gene therapy. Hum Mol Genet. 2012;21:R125-34.

14. Colombo E, Borgiani B, Verderio C, Furlan R. Microvesicles: novel biomarkers for neurological disorders. Front Physiol. 2012;3:63. https://doi.org/10.3389/fphys.2012.00063.

15. Campoy I, Lanau L, Altadill T, Sequeiros T, Cabrera S, Cubo-Abert M, Perez-Benavente A, Garcia A, Borros S, Santamaria A, Ponce J, Matias-Guiu X, Reventos J, Gil-Moreno A, Rigau M, Colas E. Exosome-like vesicles in uterine aspirates: a comparison of ultracentrifugation-based isolation protocols. J Transl Med. 2016;14:180. https://doi.org/10.1186/s12967-016-0935-4.

16. Thery C, Amigorena S, Raposo G, Clayton A. Isolation and characterization of exosomes from cell culture supernatants and 
biological fluids. In: Current Protocols in Cell Biology. Wiley; 2006. chapter 3, unit 3.22 .

17. Baranyai T, Herczeg K, Onodi Z, Voszka I, Modos K, Marton N, Nagy G, Mager I, Wood MJ, El Andaloussi S, Palinkas Z, Kumar V, Nagy P, Kittel A, Buzas EI, Ferdinandy P, Giricz Z. Isolation of exosomes from blood plasma: qualitative and quantitative comparison of ultracentrifugation and size exclusion chromatography methods. PLoS One 2015;10(12):e0145686. https://doi.org/https:// doi.org/10.1371/journal.pone.0145686.

18. Davies RT, Kim J, Jang SC, Choi EJ, Gho YS, Park J. Microfluidic filtration system to isolate extracellular vesicles from blood. Lab Chip. 2012;12:5202-10.

19. Zlotogorski-Hurvitz A, Dayan D, Chaushu G, Korvala J, Salo T, Sormunen R, Vered M. Human saliva-derived exosomes: comparing methods of isolation. J Histochem Cytochem. 2015;63:181-9.

20. Enderle D, Spiel A, Coticchia CM, Berghoff E, Mueller R, Schlumpberger M, Sprenger-Haussels M, Shaffer JM, Lader E, Skog J, Noerholm M. Characterization of RNA from exosomes and other extracellular vesicles isolated by a novel spin columnbased method. PLoS One 2015;10(8): e0136133. https://doi.org/ 10.1371/journal.pone. 0136133 .

21. Helwa I, Cai JW, Drewry MD, Zimmerman A, Dinkins MB, Khaled ML, Seremwe M, Dismuke WM, Bieberich E, Stamer WD, Hamrick MW, Liu YT. A comparative study of serum exosome isolation using differential ultracentrifugation and three commercial reagents. PLoS One 2017;12(1):e0170628. https:// doi.org/10.1371/journal.pone.0170628.

22. Stranska R, Gysbrechts L, Wouters J, Vermeersch P, Bloch K, Dierickx D, Andrei G, Snoeck R. Comparison of membrane affinity-based method with size-exclusion chromatography for isolation of exosome-like vesicles from human plasma. J Transl Med. 2018;16(1):1. https://doi.org/10.1186/s12967-017-1374-6.

23. Royo F, Zuniga-Garcia P, Sanchez-Mosquera P, Egia A, Perez A, Loizaga A, Arceo R, Lacasa I, Rabade A, Arrieta E, Bilbao R, Unda M, Carracedo A, Falcon-Perez JM. Different EV enrichment methods suitable for clinical settings yield different subpopulations of urinary extracellular vesicles from human samples. J Extracell Vesicles. 2016;5:29497. https://doi.org/10.3402/jev.v5.29497.

24. van der Pol E, Coumans F, Varga Z, Krumrey M, Nieuwland R. Innovation in detection of microparticles and exosomes. J Thromb Haemost. 2013;11:36-45.

25. van der Pol E, Coumans FA, Grootemaat AE, Gardiner C, Sargent IL, Harrison P, Sturk A, van Leeuwen TG, Nieuwland R. Particle size distribution of exosomes and microvesicles determined by transmission electron microscopy, flow cytometry, nanoparticle tracking analysis, and resistive pulse sensing. J Thromb Haemost. 2014;12:1182-92.

26. Buzas EI, Toth EA, Sodar BW, Szabo-Taylor KE. Molecular interactions at the surface of extracellular vesicles. Semin Immunopathol. 2018;40:453-64.

27. Pietrowska M, Funk S, Gawin M, Marczak L, Abramowicz A, Widlak P, Whiteside T. Isolation of exosomes for the purpose of protein cargo analysis with the use of mass spectrometry. Methods Mol Biol. 1654;2017:291-307.

28. van der Pol E, Hoekstra AG, Sturk A, Otto C, van Leeuwen TG, Nieuwland R. Optical and non-optical methods for detection and characterization of microparticles and exosomes. J Thromb Haemost. 2010;8:2596-607.

29. Havlik M, Marchetti-Deschmann M, Friedbacher G, Messner P, Winkler W, Perez-Burgos L, Tauer C, Allmaier G. Development of a bio-analytical strategy for characterization of vaccine particles combining SEC and nanoES GEMMA. Analyst. 2014;139:1412-9.

30. Engel NY, Weiss VU, Marchetti-Deschmann M, Allmaier G. nES GEMMA analysis of lectins and their interactions with glycoproteins - separation, detection, and sampling of noncovalent biospecific complexes. J Am Soc Mass Spectr. 2017;28:77-86.
31. Palviainen M, Saari H, Karkkainen O, Pekkinen J, Auriola S, Yliperttula M, Puhka M, Hanhineva K, Siljander PRM. Metabolic signature of extracellular vesicles depends on the cell culture conditions. J Extracell Vesicles. 2019;8(1):1596669. https://doi.org/10. 1080/20013078.2019.1596669.

32. Bacher G, Szymanski WW, Kaufman SL, Zollner P, Blaas D, Allmaier G. Charge-reduced nano electrospray ionization combined with differential mobility analysis of peptides, proteins, glycoproteins, noncovalent protein complexes and viruses. J Mass Spectrom. 2001;36:1038-52.

33. Kaufman SL, Skogen JW, Dorman FD, Zarrin F, Lewis KC. Macromolecule analysis based on electrophoretic mobility in air: globular proteins. Anal Chem. 1996;68:1895-904.

34. Kallinger P, Szymanski WW. Experimental determination of the steady-state charging probabilities and particle size conservation in non-radioactive and radioactive bipolar aerosol chargers in the size range of 5-40 nm. J Nanopart Res. 2015;17(4):171. https://doi. org/10.1007/s11051-015-2981-x.

35. Allmaier G, Weiss VU, Engel NY, Marchetti-Deschmann M, Szymanski WW. Soft X-ray radiation applied in the analysis of intact viruses and antibodies by means of nano electrospray differential mobility analysis. In: Banoub J, Caprioli R, editors: Molecular technologies for detection of chemical and biological agents. NATO Science for Peace and Security Series A: Chemistry and Biology. Dordrecht: Springer; 2017 pp. 149-157.

36. Weiss VU, Frank J, Piplits K, Szymanski WW, Allmaier G. Bipolar corona discharge-based charge equilibration for nano electrospray gas-phase electrophoretic mobility molecular analysis of bio- and polymer nanoparticles. Anal Chem. 2020;92:8665-9.

37. Chen DR, Pui DYH, Hummes D, Fissan H, Quant FR, Sem GJ. Design and evaluation of a nanometer aerosol differential mobility analyzer (Nano-DMA). J Aerosol Sci. 1998;29:497-509.

38. Flagan RC. History of electrical aerosol measurements. Aerosol Sci Technol. 1998;28:301-80.

39. Elzey S, Tsai DH, Yu LL, Winchester MR, Kelley ME, Hackley VA. Real-time size discrimination and elemental analysis of gold nanoparticles using ES-DMA coupled to ICP-MS. Anal Bioanal Chem. 2013;405:2279-88.

40. Carazzone C, Raml R, Pergantis SA. Nanoelectrospray ion mobility spectrometry online with inductively coupled plasma-mass spectrometry for sizing large proteins, DNA, and nanoparticles. Anal Chem. 2008;80:5812-8.

41. Shah VB, Orf GS, Reisch S, Harrington LB, Prado M, Blankenship RE, Biswas P. Characterization and deposition of various lightharvesting antenna complexes by electrospray atomization. Anal Bioanal Chem. 2012;404:2329-38.

42. Weiss VU, Urey C, Gondikas A, Golesne M, Friedbacher G, von der Kammer F, Hofmann T, Andersson R, Marko-Varga G, Marchetti-Deschmann M, Allmaier G. Nano electrospray gasphase electrophoretic mobility molecular analysis (nES GEMMA) of liposomes: applicability of the technique for nano vesicle batch control. Analyst. 2016;141:6042-50.

43. Chernyshev VS, Rachamadugu R, Tseng YH, Belnap DM, Jia Y, Branch KJ, Butterfield AE, Pease LF 3rd, Bernard PS, Skliar M. Size and shape characterization of hydrated and desiccated exosomes. Anal Bioanal Chem. 2015;407:3285-301.

44. Allmaier G, Laschober C, Szymanski WW. Nano ES GEMMA and PDMA, new tools for the analysis of nanobioparticles - protein complexes, lipoparticles, and viruses. J Am Soc Mass Spectr. 2008;19:1062-8.

45. Weiss VU, Balantic K, Pittenauer E, Tripisciano C, Friedbacher G, Weber V, Marchetti-Deschmann M, Allmaier G. Nano electrospray differential mobility analysis based size-selection of liposomes and very-low density lipoprotein particles for offline hyphenation to MALDI mass spectrometry. J Pharmaceut Biomed. 2020;179:112998. 
46. Tripisciano C, Weiss R, Eichhorn T, Spittler A, Heuser T, Fischer MB, Weber V. Different potential of extracellular vesicles to support thrombin generation: contributions of phosphatidylserine, tissue factor, and cellular origin. Sci Rep-UK. 2017;7(1):6522. https:// doi.org/10.1038/s41598-017-03262-2.

47. Weiss R, Groger M, Rauscher S, Fendl B, Eichhorn T, Fischer MB, Spittler A, Weber V. Differential interaction of platelet-derived extracellular vesicles with leukocyte subsets in human whole blood. Sci RepUK. 2018;8(1):6598. https://doi.org/10.1038/s41598-018-25047-X.

48. George SK, Lauková L, Weiss R, Semak V, Fendl B, Weiss VU, Steinberger S, Allmaier G, Tripisciano C, Weber V. Comparative analysis of platelet-derived extracellular vesicles using flow cytometry and nanoparticle tracking analysis. Int J Mol Sci. 2021;22(8):3839-48.

49. Tycova A, Prikryl J, Foret F. Reproducible preparation of nanospray tips for capillary electrophoresis coupled to mass spectrometry using 3D printed grinding device. Electrophor. 2016;37: 924-30.

50. Shevchenko A, Wilm M, Vorm O, Mann M. Mass spectrometric sequencing of proteins silver-stained polyacrylamide gels. Anal Chem. 1996;68(5):850-8.

51. Shevchenko A, Tomas H, Havlis J, Olsen JV, Mann M. In-gel digestion for mass spectrometric characterization of proteins and proteomes. Nat Protoc. 2006;1:2856-60.

52. Cox J, Mann M. MaxQuant enables high peptide identification rates, individualized p.p.b.-range mass accuracies and proteomewide protein quantification. Nat Biotechnol. 2008;26:1367-72.

53. Cox J, Neuhauser N, Michalski A, Scheltema RA, Olsen JV, Mann M. Andromeda: a peptide search engine integrated into the MaxQuant environment. J Proteome Res. 2011;10:1794-805.

54. Tyanova S, Temu T, Cox J. The MaxQuant computational platform for mass spectrometry-based shotgun proteomics. Nat Protoc. 2016;11:2301-19.

55. Tyanova S, Temu T, Sinitcyn P, Carlson A, Hein MY, Geiger T, Mann M, Cox J. The Perseus computational platform for comprehensive analysis of (prote)omics data. Nat Methods. 2016;13:731-40.

56. Perez-Riverol Y, Csordas A, Bai JW, Bernal-Llinares M, Hewapathirana S, Kundu DJ, Inuganti A, Griss J, Mayer G, Eisenacher M, Perez E, Uszkoreit J, Pfeuffer J, Sachsenberg T, Yilmaz S, Tiwary S, Cox J, Audain E, Walzer M, et al. The PRIDE database and related tools and resources in 2019: improving support for quantification data. Nucleic Acids Res. 2019;47: D442-50.

57. Urey C, Weiss VU, Gondikas A, von der Kammer F, Hofmann T, Marchetti-Deschmann M, Allmaier G, Marko-Varga G, Andersson R. Combining gas-phase electrophoretic mobility molecular analysis (GEMMA), light scattering, field flow fractionation and cryo electron microscopy in a multidimensional approach to characterize liposomal carrier vesicles. Int J Pharm. 2016;513:309-18.

58. Weiss VU, Wieland K, Schwaighofer A, Lendl B, Allmaier G. Native nano-electrospray differential mobility analyzer (nES GEMMA) enables size selection of liposomal nanocarriers combined with subsequent direct spectroscopic analysis. Anal Chem. 2019;91:3860-8.

59. Wieland K, Ramer G, Weiss VU, Allmaier G, Lend1 B, Centrone A. Nanoscale chemical imaging of individual chemotherapeutic cytarabine-loaded liposomal nanocarriers. Nano Res. 2019;12: 197-203.

60. Palviainen M, Saraswat MK, Varga Z, Kitka D, Neuvonen M, Puhka M, Joenvaara S, Renkonen R, Nieuwland R, Takatalo M, Siljander PRM. Extracellular vesicles from human plasma and serum are carriers of extravesicular cargo-implications for biomarker discovery. PLoS One 2020;15(8): e0236439. https://doi.org/10. 1371/journal.pone. 0236439 .

61. Tutanov O, Proskura K, Kamyshinsky R, Shtam T, Tsentalovich Y, Tamkovich S. Proteomic profiling of plasma and total blood exosomes in breast cancer: a potential role in tumor progression, diagnosis, and prognosis. Front Oncol. 2020;10:2173. https://doi. org/10.3389/fonc. 2020.580891 .

Publisher's note Springer Nature remains neutral with regard to jurisdictional claims in published maps and institutional affiliations.

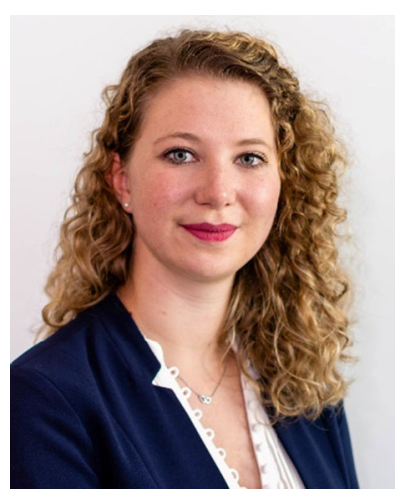

Stephanie Steinberger is a $\mathrm{PhD}$ student in the research group "Mass Spectrometric Bio and Polymer Analytics" at TU Wien, Institute of Chemical Technologies and Analytics, Vienna, Austria. Her current project focuses on method development for the characterization of extracellular vesicles via nano electrospray gas-phase electrophoresis (nES GEMMA) and mass spectrometry (MS).

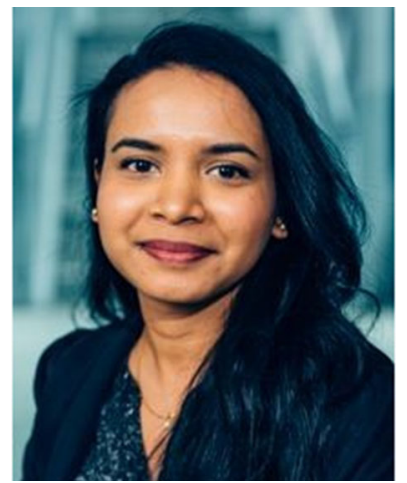

Sobha Karuthedom George is a doctoral candidate in the Center for Biomedical Technology at Danube University Krems, Austria. Her research interests lie in uncovering the role of extracellular vesicles $(\mathrm{EVs})$ in different physiological and pathological settings.

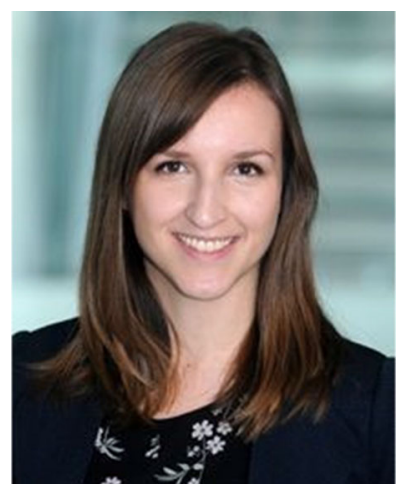

Lucia Lauková finished her $\mathrm{PhD}$ at the Institute of Molecular Biomedicine, Faculty of Medicine, Comenius University in Bratislava, Slovakia. Since 2018, she has been a postdoctoral fellow at the Center for Biomedical Technology at Danube University Krems, Austria. Her research interests are extracellular DNA, NETosis, and extracellular vesicles (EVs). 


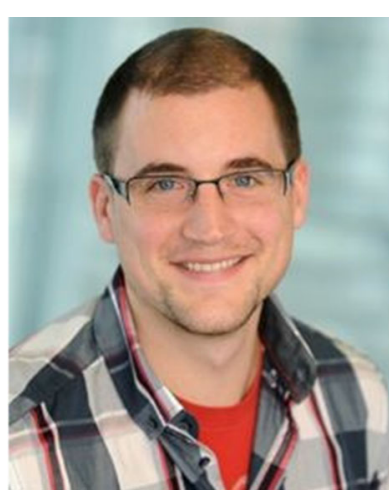

René Weiss studied molecular biology at the University of Vienna, Austria, and received his $\mathrm{PhD}$ in immunology from the Medical University of Vienna, Austria, in 2013. Since 2013, he has been conducting research in the field of sepsis at the Center for Biomedical Technology at Danube University Krems, Austria, and is specialized in the topic of extracellular vesicles (EVs).

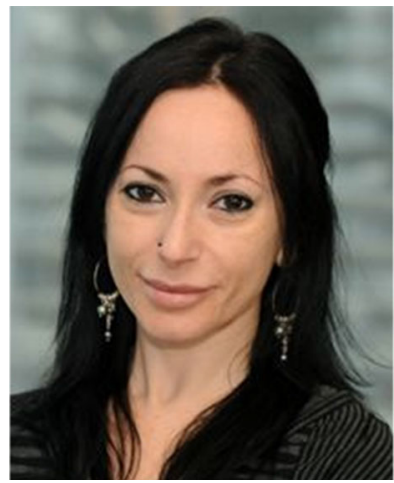

Carla Tripisciano is Assistant Professor in Biomaterials and Blood-Material Interactions at the Center for Biomedical Technology, Department for Biomedical Research, Danube University Krems, Austria. She has been working for several years on the immunological and procoagulant activity of blood cellderived extracellular vesicles and the application of extracellular vesicles as diagnostic markers in pathologies with increased thrombotic risk. She serves on the advisory board of the Austrian Society for Extracellular Vesicles (ASEV).

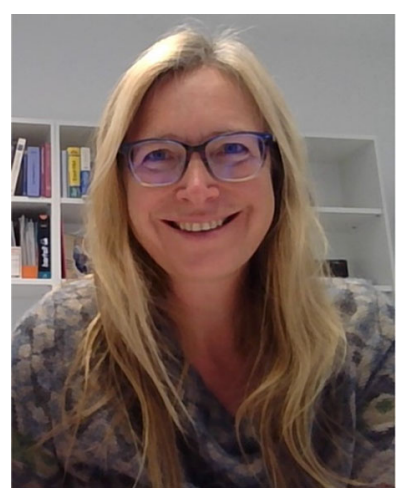

Ruth Birner-Gruenberger holds professorships for bioanalytics and biochemistry at TU Wien, Institute of Chemical Technologies and Analytics, Vienna, Austria, and Medical University of Graz, Austria. She is an expert in lipid metabolism and mass spectrometry-based omics technologies.

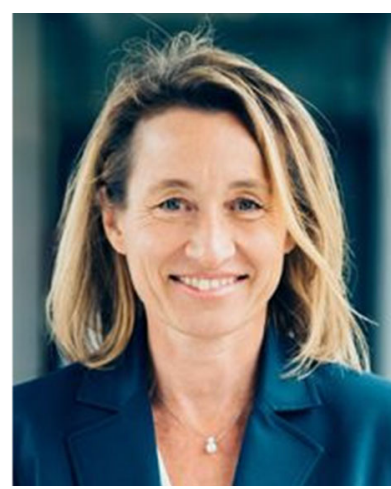

Viktoria Weber is Head of the Department for Biomedical Research and Full Professor for Medical Biochemistry at Danube University Krems, Austria. She is President of the European Society for Artificial Organs and cofounder of the Austrian Society for Extracellular Vesicles (ASEV). Her research focuses on the blood-biomaterial interface, the activation of blood cells during blood-material contact, and extracellular vesicles (EVs) and their roles in coagulation and immunomodulation, particularly in the setting of inflammation and sepsis.

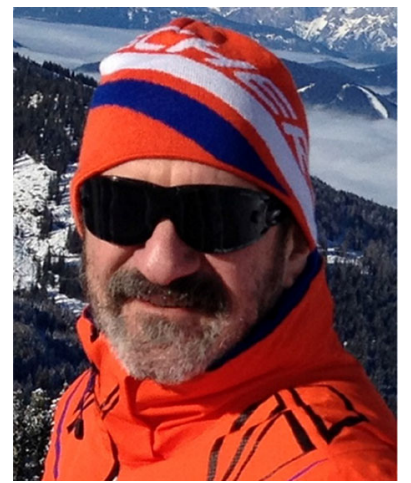

Günter Allmaier is a graduate from the University of Vienna (M. Pharm. Sc. and Ph.D.). Currently, he is Full Professor of Analytical Chemistry at the Institute of Chemical Technologies and Analytics (TU Wien, Vienna, Austria). He has been working since the beginning of the 1980s in the field of mass spectrometry and analysis of bioactive compounds with emphasis on therapeutic proteins and proteomics/lipidomics/glycomics. Furthermore, he was working in the field of intact cell mass spectrometry for microorganism identification and introduced different mass spectrometric techniques into the field of tribology. During the last 20 years, he has also been engaged in the field of electrophoretic separation in the liquid and gas phase (gas-phase electrophoretic molecular mobility analysis) of various classes of bioaggregates, vaccine particles, and nano(bio)particles.

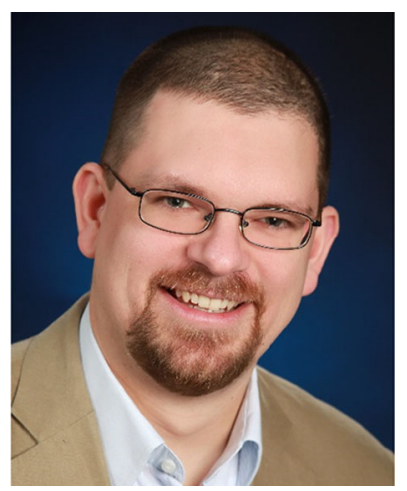

Victor U. Weiss is Assistant Professor in the research group "Mass spectrometric Bio and Polymer Analytics" at TU Wien, Institute of Chemical Technologies and Analytics, Vienna, Austria. In his research, he focusses on the characterization and detection of nanoparticle material (organic, inorganic, and biological, e.g., liposomes, viruses, and virus-like particles) by application of electrophoretic techniques (liquid-phase electrophoresis in the capillary and the chip format as well as gas-phase electrophoresis). Furthermore, he works on the on- and offline hyphenation of gas-phase electrophoresis to orthogonal analysis methods. 